Journal of the

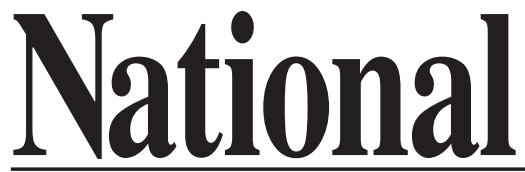

Academy or

Forensic
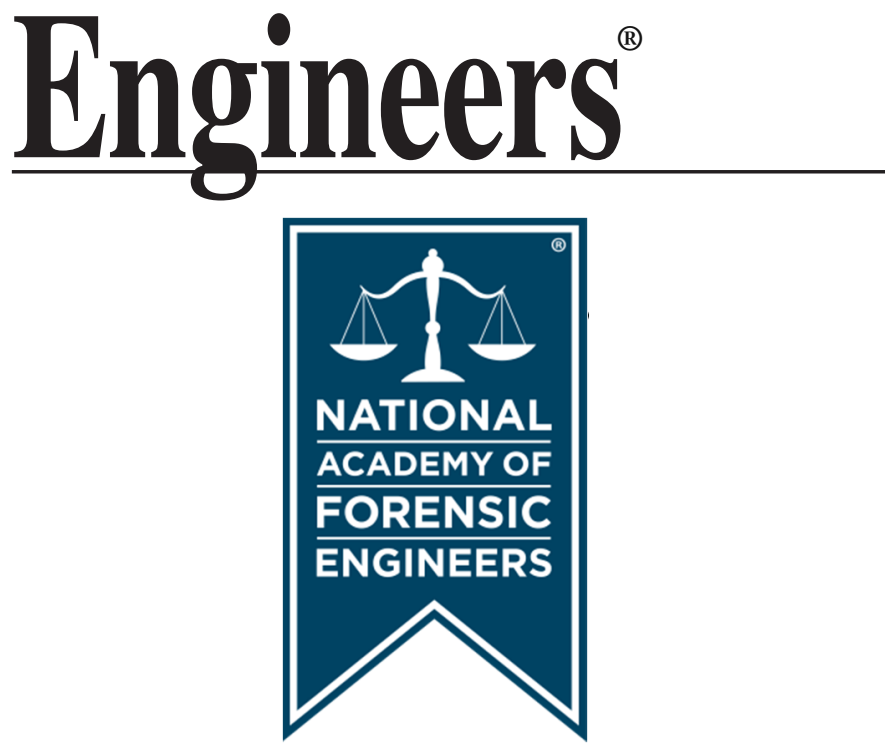

http://www.nafe.org ISSN: 2379-3252 


\title{
Forensic Engineering Report: Finite Element Analysis of a Flex-Fan Blade Failure
}

by John Marcosky, P.E. (NAFE 205F)

\begin{abstract}
Dynamic failure of an after-market automotive radiator cooling flex-fan will be used to describe the application of finite element analysis as a Forensic Engineering diagnostic and reconstruction tool. The replacement flex-fan assembly installation and operation history prior to the failure event will be presented including methodologies employed during the reconstruction.
\end{abstract}

\section{Introduction}

During normal use, mechanical mechanisms may experience dynamic loads not identified during the design process or pre production testing. Laboratory static tests quite frequently do not address dynamic loads and subsequent com ponent stress distribution. Components such as a vehicle engine driven radiator cooling flex-fan are designed to change shape during their operating range and therefore experience unidentified increased stress areas.

A recently installed replacement automotive engine driven cooling flex-fan experienced attachment rivet failures and several blade separations from the companion hub assembly. Reconstruction and analysis were conducted using accepted engineering principals. These principals include; anticipated dynamic fan blade loads, material specifications, stress analysis of component parts, Optical Microscopic, "O.M." and Scanning Electron Microscopic, "S.E.M." analysis. Dynamic flex-fan blade deformation and stress distribution experi enced by the fan blade were observed using a finite element analysis. This anal ysis revealed flex-fan blade resonant frequencies, corresponding blade flutter and high concentrated stress at the attachment rivet areas throughout the engine rpm operating range.

\section{Accident Background}

Prior to this accident, a general engine tune up of a 1970 Chevrolet Camaro equipped with a 350 cubic inch V-8 was performed at the owners residence. Included was replacement of the existing electric radiator cooling fan with an aftermarket engine driven flex-fan. This fan required a 2-inch spacer between the existing water pump and fan hub for proper radiator surface orientation. All 
Copyright @ National Academy of Forensic Engineers (NAFE) http://www.nafe.org. Redistribution or resale is illegal. Originally published in the Journal of the NAFE volume indicated on the cover page. ISSN: 2379-3252

fan attachment bolts were tightened, the $\mathrm{V}$ belt installed and checked for adjustment. A stationary vehicle open hood inspection was conducted with the engine running at idle to verify proper pulley and fan alignment. This vehicle was not equipped with a radiator-fan shroud. The Camaro was then driven approximately twenty-five miles on city streets and returned to the residence.

Following this test drive an open hood, engine running examination was conducted during which the throttle linkage was blipped three times. Estimated peak engine speed was between four and five thousand rpm. The operator was standing adjacent to the vehicle left front fender facing the engine compartment. His right hand was extended to the carburetor throttle linkage and left-hand resting on the fender. During the last rev cycle, three adjacent flex-fan blades separated from the hub assembly. One struck the operator in the face and another penetrated the open hood panel as shown in photo \#1 and 2. A third struck an adjacent car.

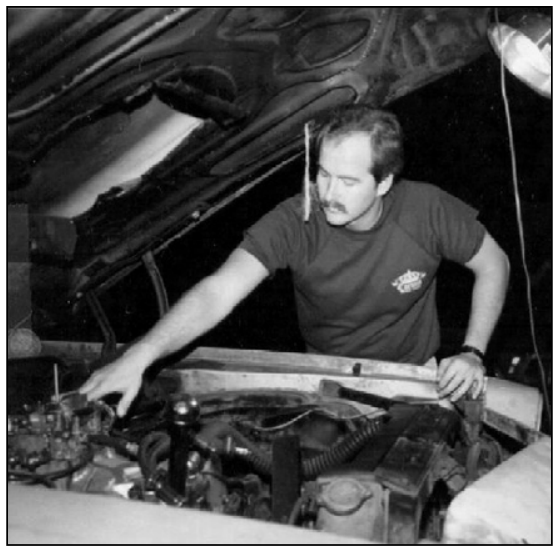

Photo 1

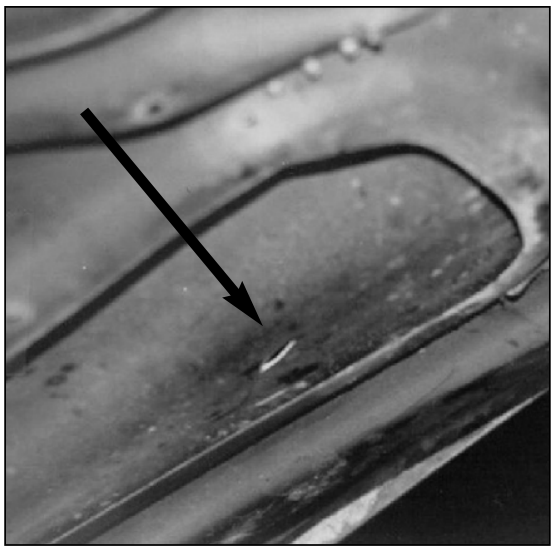

Photo 2

The operator lost his left eye, fifteen percent of his frontal lobe, partial loss of hearing and balance. Witness statements indicate due care was performed during the fan installation and this incident.

\section{Vehicle Inspection}

A post event inspection of the subject 1970 Camaro engine bay was conducted at the residence where it was preserved in its after accident condition. The original flex-fan assembly consists of a steel spider-shaped hub with two identical, painted, six-point legs that sandwich the fan blades. Three aluminum rivets for each blade or a total of eighteen rivets retain the complete assembly. Four equally spaced bolt mounting holes are located at the hub center area which does not have an internal spacer to compensate for blade thickness. This installation required a 2 -inch spacer between the fan hub and water pump pulley. 
Copyright @ National Academy of Forensic Engineers (NAFE) http://www.nafe.org. Redistribution or resale is illegal. Originally published in the Journal of the NAFE volume indicated on the cover page. ISSN: 2379-3252

Examination of the engine bay revealed the replacement fan hub assembly and two inch spacer were properly attached to the water pump pulley. This vehicle was not equipped with a radiator fan shroud as shown in photo \#3 and \#4.

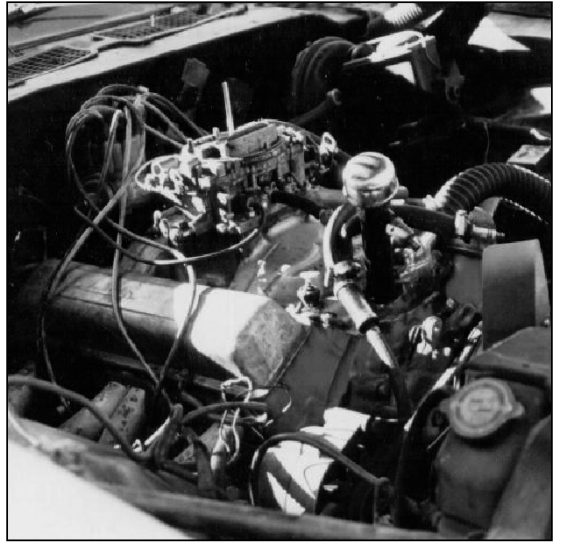

Photo 3

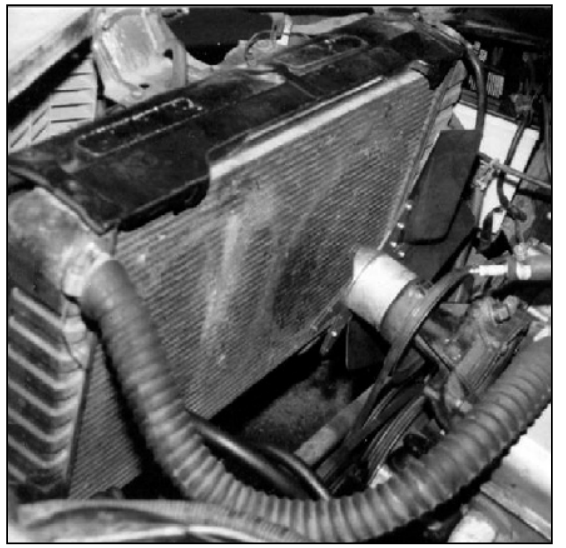

Photo 4

Each hub spider leg and corresponding remaining fan blade was assigned a reference number for photographic identification purposes. Three stainless steel blades remained attached to the steel fan hub spider by their aluminum rivets as shown in photo $\# 5$ and 6 . Blade numbers 4 and 6 were loose at their rivet attachments and could be rotated at the outer edge approximately $1 / 32$ inch.

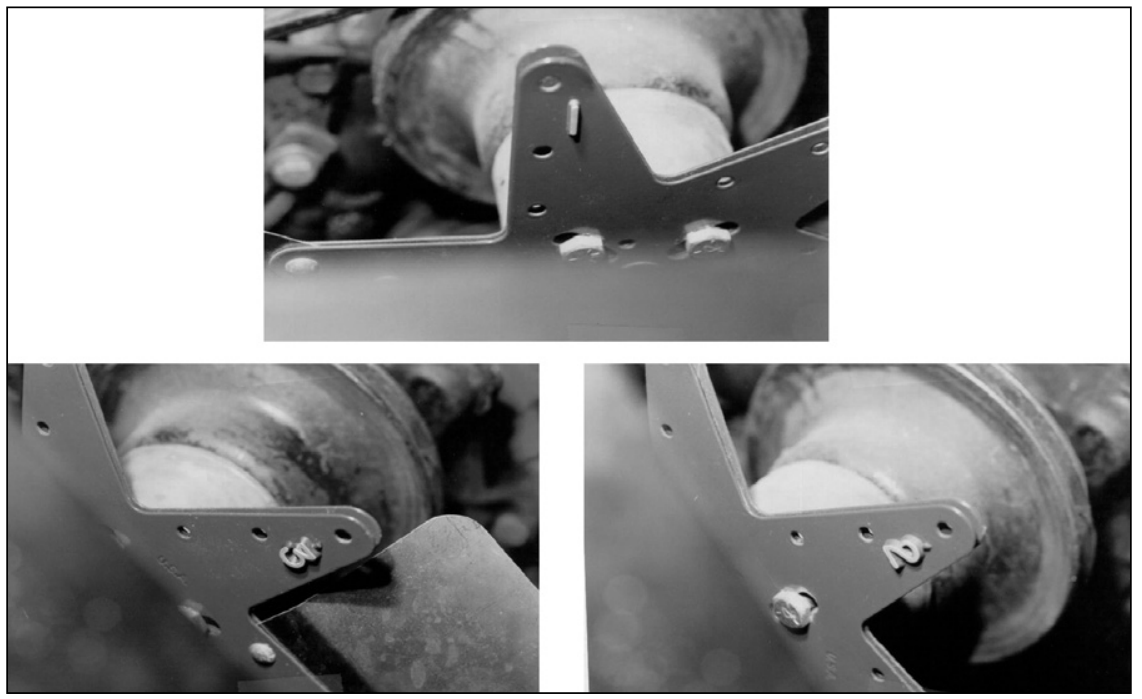

Photo 5 


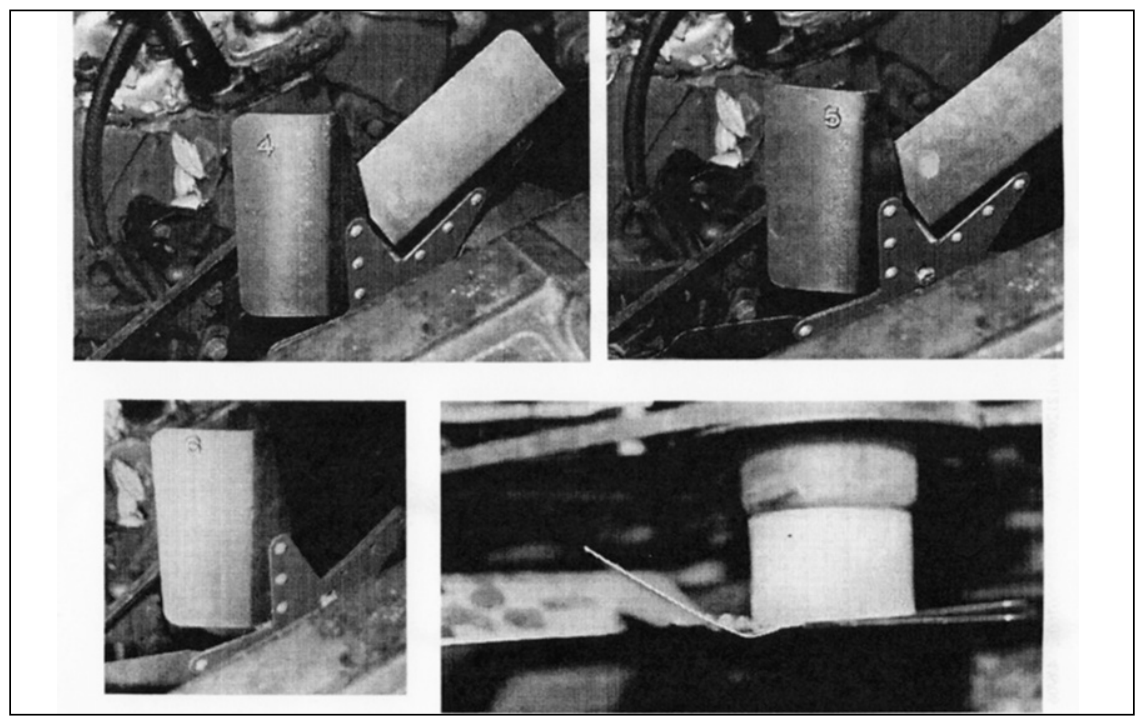

\section{Photo 6}

Inspection of the hub rivet holes for each separated fan blade revealed no oval distortion or exterior paint disturbance. Both hub faces were spread apart at these locations. Blade number six was bent about a radial axis toward the radiator.

Engine pulley outside diameter measured $73 / 4$ inches and the water pump pulley measured $61 / 4$ inches. Both pulleys were in alignment and the water pump shaft appeared unaffected by this event. Fan belt tension was normal. No additional engine compartment damage was observed.

Three detached highly deformed fan blades, five manufactured rivet head segments and seven formed (bucked) rivet end segments were recovered from the scene as shown in Photo \#7.

Reportedly, no other rivet segments were found at the accident scene. The fan blades are stainless steel and measured 0.027 inch-thick. Hub assembly (two spiders and the blade) thickness mea sured about 0.103

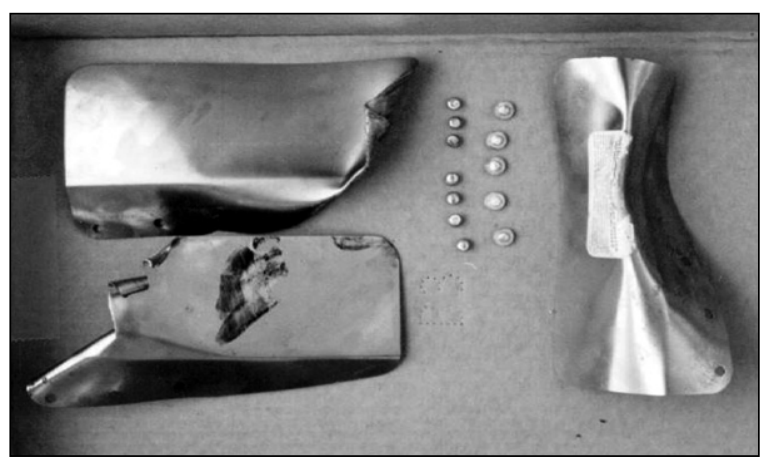

Photo 7 
Copyright @ National Academy of Forensic Engineers (NAFE) http://www.nafe.org. Redistribution or resale is illegal. Originally published in the Journal of the NAFE volume indicated on the cover page. ISSN: 2379-3252

inches. The convex side of one blade bore a notice that this side was to be installed toward the radiator. Each detached blade was assigned an arbitrary number, one to three for identification; not intended to match a pre event spider location.

\section{Flex-Fan Blade Failure Analysis}

An exemplar fan, Photo \#8, was obtained which had six 0.027 -inch-thick stainless steel blades, each fastened between two painted steel spiders (that formed the hub) by three aluminum rivets. The hub assembly (two spiders and the blade thickness) was about 0.103 inches thick. These stainless steel blades were moderately magnetic, indicating that they had been cold worked: their hardness was Rockwell 15N 77.4 (equivalent to an ultimate tensile strength of approximately $152,000 \mathrm{psi}$. The convex side of one blade bore a notice that this side was to be

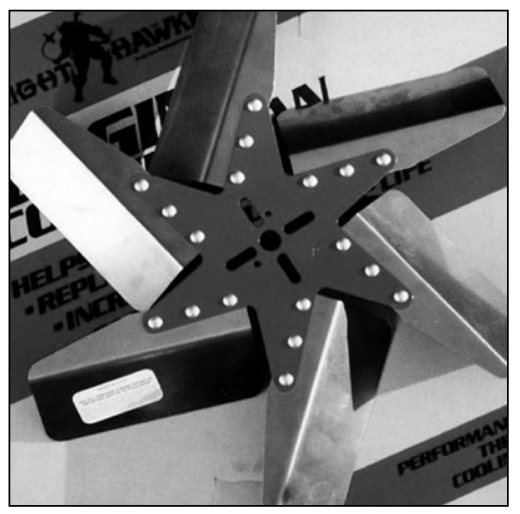

Photo 8 installed toward the radiator.

Rivet bucked end measurements varied from 0.233 to 0.249 inches in diam eter; manufactured heads were about 0.4 inches in diameter. Hardness tests were attempted on the flat surface of the bucked rivet ends; however, because these ends were not perfectly flat and perpendicular to the rivet axis, the data obtained were not reproducible and therefore invalid.

\section{Separated Blades From the Subject Flex-Fan}

The three detached fan blades to be examined were numbered for purposes of identification; fan blades 1, 2 and 3 and are shown in Photo \# 9. For ease of reference, the two sides of each blade were called the concave (cc) and convex (cx), respectively; rivet holes were identified as the inner $(\mathrm{I})$, center $(\mathrm{C})$ and outer $(\mathrm{O})$, referring to their distance from the center of the fan assembly. Blades 1 and 2 exhibited possible evidence of multiple strikes. Blade 3 did not exhibit as severe a damage pattern, but had

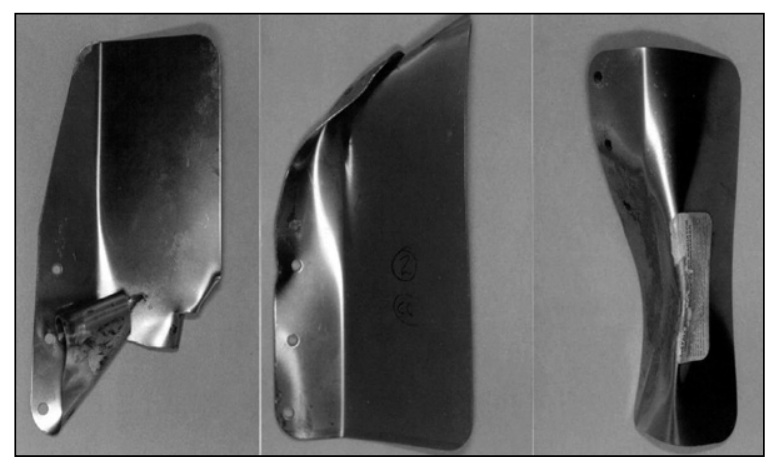

Photo 9 
Copyright @ National Academy of Forensic Engineers (NAFE) http://www.nafe.org. Redistribution or resale is illegal. Originally published in the Journal of the NAFE volume indicated on the cover page. ISSN: 2379-3252

been "pinched" at a point just beyond the tip of the spider. Blade 3 also had the installation-information note attached.

\section{Separated Blades Rivet Holes}

Both sides of each of the rivet holes on the three blades were examined under binocular microscope. On all of the holes, the surface surrounding the convex side had a rubbed appearance, whereas the concave side appeared smooth with a rounded edge. As both sides of the blades are in contact with the steel spider interior, the cause of this difference in appearance is believed to be the hole punching process. Rivet holes were found to have a diameter of about 0.185 inch.

None of the holes appeared distorted or out-of-round; however, the edges of several holes appeared to have "lips" or roughness on the convex side and therefore may also be related to the hole forming process. However, in a few cases, a "lip" was observed on the concave side. In these cases, the "lip" is believed to be related to the separation incident.

Scanning electron microscope photographs were also taken of several blade holes. A halo appearance was observed on the concave side and appears to be part of the hole punching process. These examinations confirmed the results of the optical examination.

\section{Failed Rivet Examinations:}

Four of the five failed manufactured rivet head segments and six of the seven failed bucked rivet end segments recovered at the scene shown on photo \#10 were available for this examination. Reportedly, no other rivet pieces were found at the accident scene.

\section{Failure of the nine rivets} used to assemble the three blades to the spiders would be expected to result in a total of eighteen rivet pieces, there are apparently six rivet pieces missing. Available samples may represent

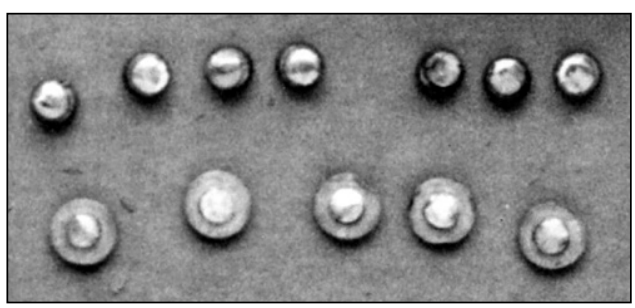

Photo 10 as few as six of the nine rivets (if all four manufactured heads were originally attached to four of the six bucked ends) or as many as all nine of the rivets. No attempt was made to match the various manufactured heads with their corre sponding bucked ends. Research revealed rivets used by the manufacturer were heat treated aluminum 5052 alloy. Rivet dimensions, material specifications and strength characteristics were obtained from engineering drawings. 
Copyright @ National Academy of Forensic Engineers (NAFE) http://www.nafe.org. Redistribution or resale is illegal. Originally published in the Journal of the NAFE volume indicated on the cover page. ISSN: 2379-3252

A mathematical analysis during the engine operating range, revealed insufficient centrifugal forces acting on the blades which would produce rivet overload stress conditions and subsequent failure. The results are presented in the finite element analysis section.

Because they were deformed by the separation process, as well as by the original riveting process, the diameters of the rivet shanks were difficult to accurately measure; however, their diameters appeared to vary between 0.182 and 0.183 inches.

Optical photographs of the rivet fracture surfaces are shown on photos \#11, $\# 12$ and \#13. All of the fracture surfaces are relatively flat, perpendicular to the rivet axis, and composed of two different appearing areas on the fracture surface: a large, shiny, rubbed area and a small, dull-appearing, area. The dull area on all the rivet pieces were found to be composed of "dimples". "Dimple" is a term used to describe the details of the fracture surface generated by microvoid coalescence, which is the characteristic mode of failure of ductile metals when they have been subject to loads in excess of their strength. In addition, the shape of the "dimples" relates to the direction at which the loads were applied relative to the fracture surface. For example; on the fracture surface of these rivets, the shape of the "dimples" corresponds to shear fracture. The general deformation of the rivet segments supports the finding of a rivet shear failure. The shiny area will be described during the analysis of photo \#14.

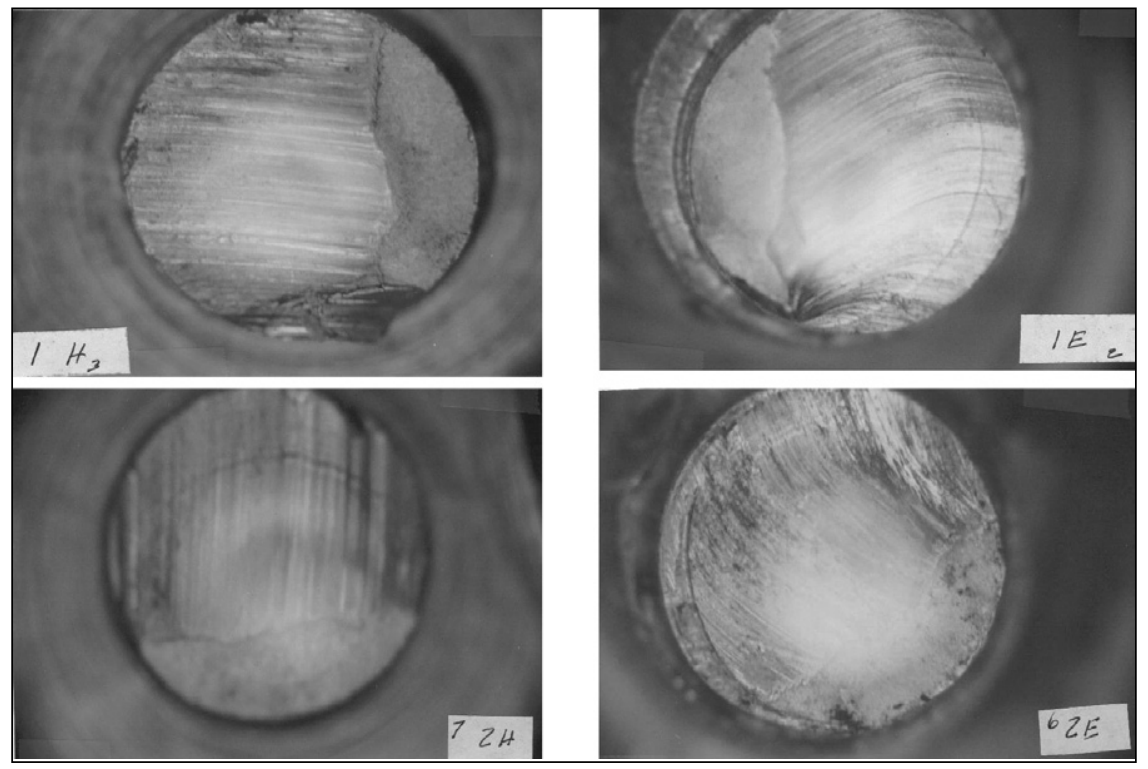


Copyright @ National Academy of Forensic Engineers (NAFE) http://www.nafe.org. Redistribution or resale is illegal. Originally published in the Journal of the NAFE volume indicated on the cover page. ISSN: 2379-3252

No evidence of a typical prior fatigue crack, any other sub-critical crack growth, or any material or manufacturing defect was found on any of the rivet segments. Fatigue striations or "beach" marks would be a concentric ring pattern emanating from the origin site of the fatigue crack and were not observed both optically and in the scanning electron microscope. Additionally, the detailed

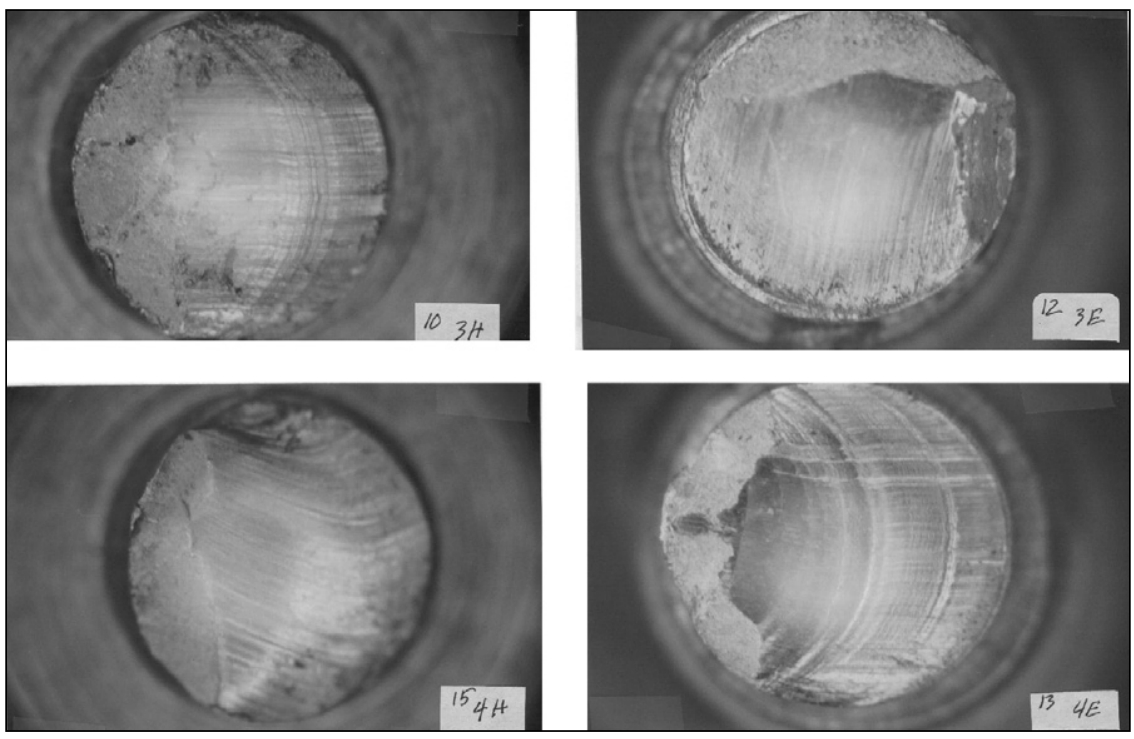

Photo 12

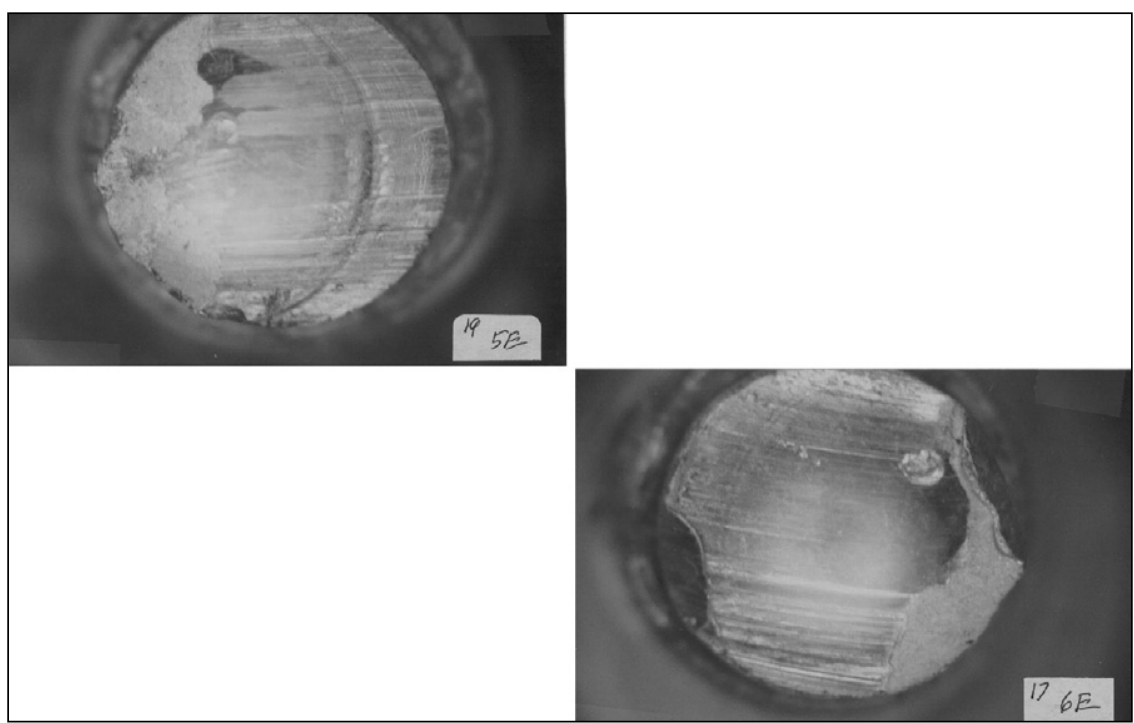


Copyright @ National Academy of Forensic Engineers (NAFE) http://www.nafe.org. Redistribution or resale is illegal. Originally published in the Journal of the NAFE volume indicated on the cover page. ISSN: 2379-3252

structure of the dull portion of the rivet showed that deformation had occurred, and in the fracture failure the forces involved were usually well below the yield stress of the material in shear. Therefore, deformation does not occur except in the last area to fail and would be limited in scope. In these rivets, there exhibited extensive evidence of deformation.

Shiny fracture surface of rivet $(3 \mathrm{H})$ reveals both straight and semicircular lines leading to a final fracture surface; shown on photo \# 14. The straight lines are shear and the semicircular are chatter marks or "chatter band" where the shear forma tion occurred. These chatter marks are part of

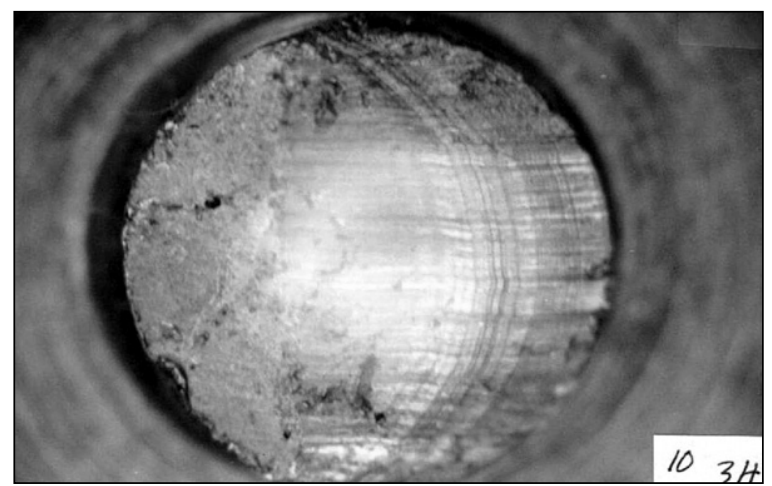

Photo 14

a concentric circle and appear formed by the outside surface of a hole as it chattered, sheared and was pulled across the fracture surface. Chatter marks differ from fatigue marks which would be concentric around a point of origin, instead they are concentric about a point more or less in the center of the shank. Additionally, fatigue beach marks would have to predate the shearing process and the shearing process would obliterate them. Therefore, these marks had to be created at the same time as the shearing process in order to still be in existence and therefore are not fatigue beach marks.

Considering the short flex-fan blade assembly operational time period and subsequent blade separations combined with insufficient calculated overload forces acting on the blade attachment rivets; a finite element analysis was conducted to determine if unknown blade configurations and stress distributions existed during dynamic conditions.

\section{Finite Element Analysis of the Flex-Fan Blade:}

The finite element method is a numerical analysis procedure for obtaining an appropriate solution to engineering problems that do not have exact mathematical solutions. It is a very diverse and flexible tool and can be applied to a broad range of fields from stress analysis to acoustic and flow problems includ ing static, dynamic and thermal analysis. In the static analysis, the computer determines the element stresses and deflections. The eigenvalues, eigenvectors or natural frequencies and node shapes are computed in the dynamic analysis. 
Copyright @ National Academy of Forensic Engineers (NAFE) http://www.nafe.org. Redistribution or resale is illegal. Originally published in the Journal of the NAFE volume indicated on the cover page. ISSN: 2379-3252

In the finite element method of structural analysis, the part or structure is broken down into a series of discrete or "finite" volumes of the part material These are called elements, and they may be rectangular or triangular for 2-D analysis or polyhedrons for 3-D models. The geometric points defining the element corner points are called nodes. Behavior of the finite element model will converge to the behavior of the real structure if proper modeling and analysis techniques are used. The part loading is simulated by applying proper force magnitude to the nodes. Restraints to the part are also imposed at specified nodal points. Celestial Software Inc., Images 3D Finite Element Analysis program and Interactive Microcomputer Analysis \& Graphics of Engineering Systems were used for this analysis. Blade mesh, element and node identifications are shown in Figure \#1, \#2 and \#3.

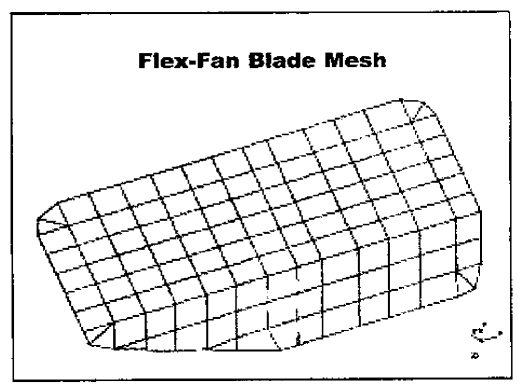

Figure 1
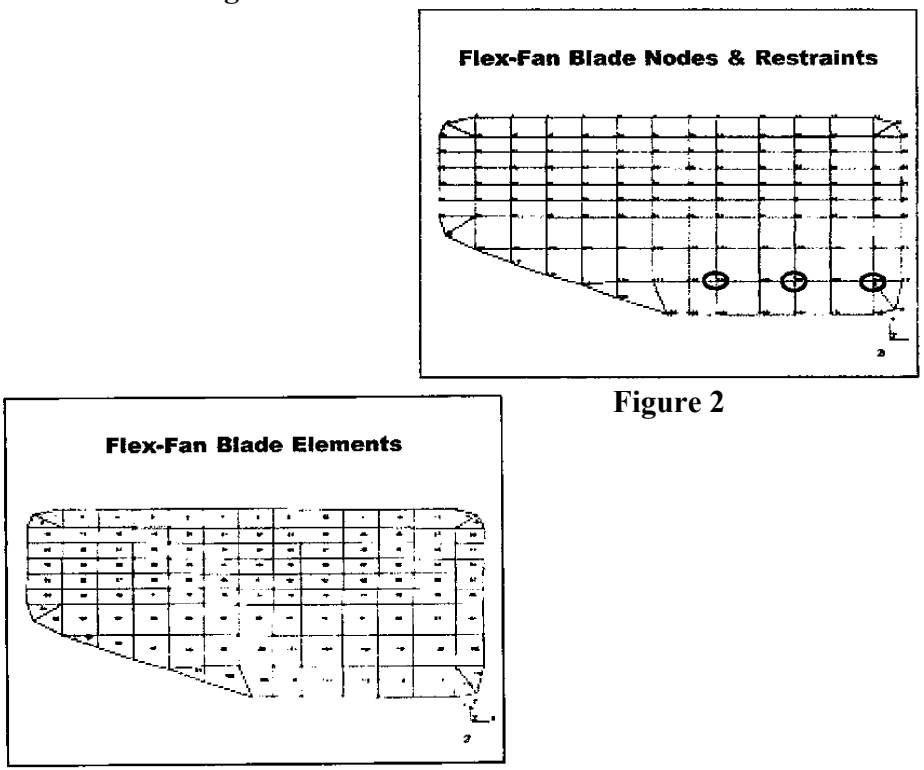

Figure 2

Figure 3 
Copyright @ National Academy of Forensic Engineers (NAFE) http://www.nafe.org. Redistribution or resale is illegal. Originally published in the Journal of the NAFE volume indicated on the cover page. ISSN: 2379-3252

The following three load case flex-fan blade stress conditions are presented in the finite element analysis:

1. Fan blade centrifugal force at $400 \mathrm{rad} . / \mathrm{sec}$.

2. 0.20 psi axial pressure differential

3. Combination of axial pressure differential and centrifugal force

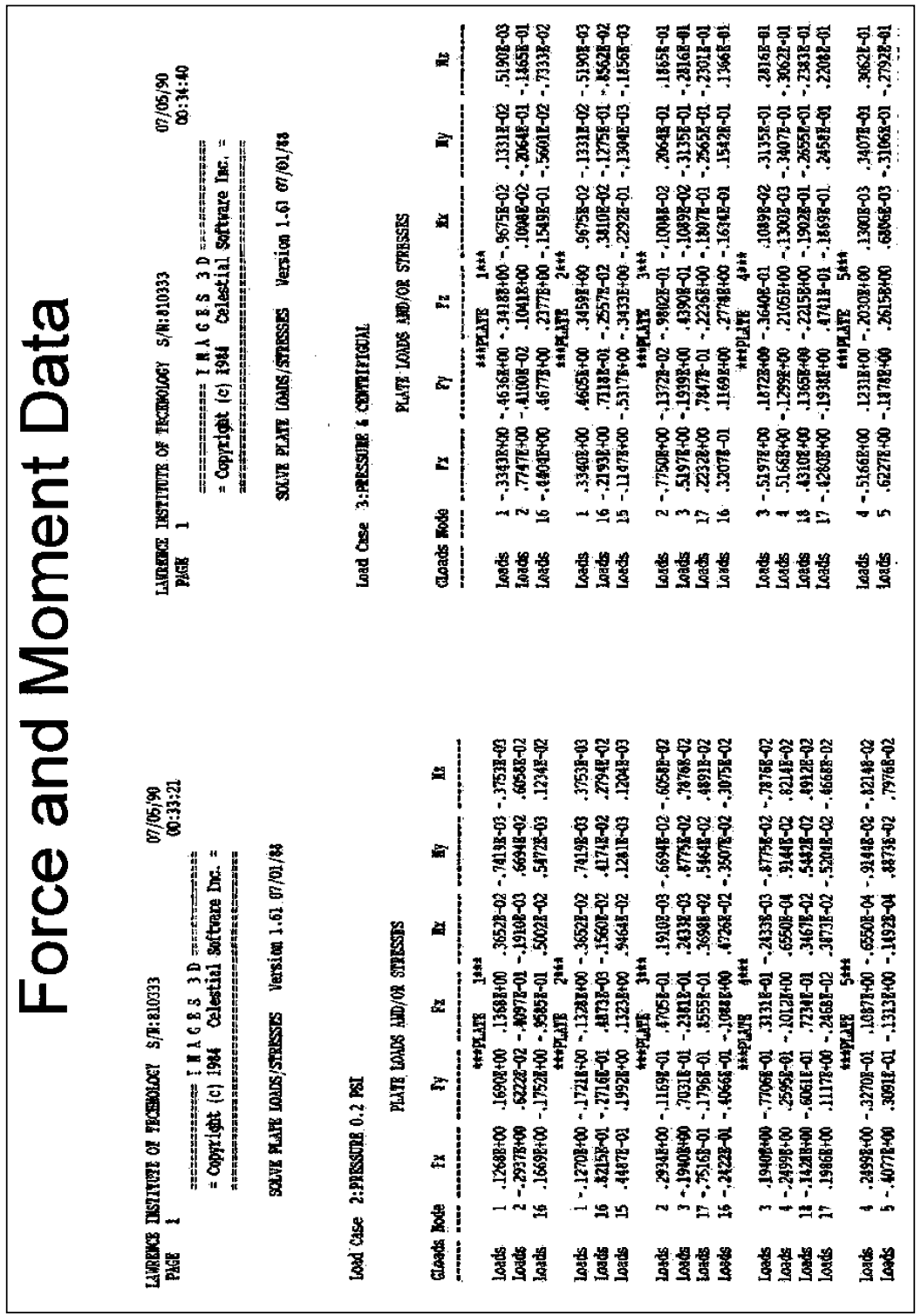

Figure 4 
Copyright $\odot$ National Academy of Forensic Engineers (NAFE) http://www.nafe.org. Redistribution or resale is illegal. Originally published in the Journal of the NAFE volume indicated on the cover page. ISSN: 2379-3252

Figure \#4 is a partial tabulation of the flex-fan blade Force and Moment data for the axial pressure differential and combination pressure differential and centrifugal force.

Figure \#5 is a partial tabulation of the flex-fan blade plate Element Nodal Principal Stress for the combination of pressure differential and centrifugal force.

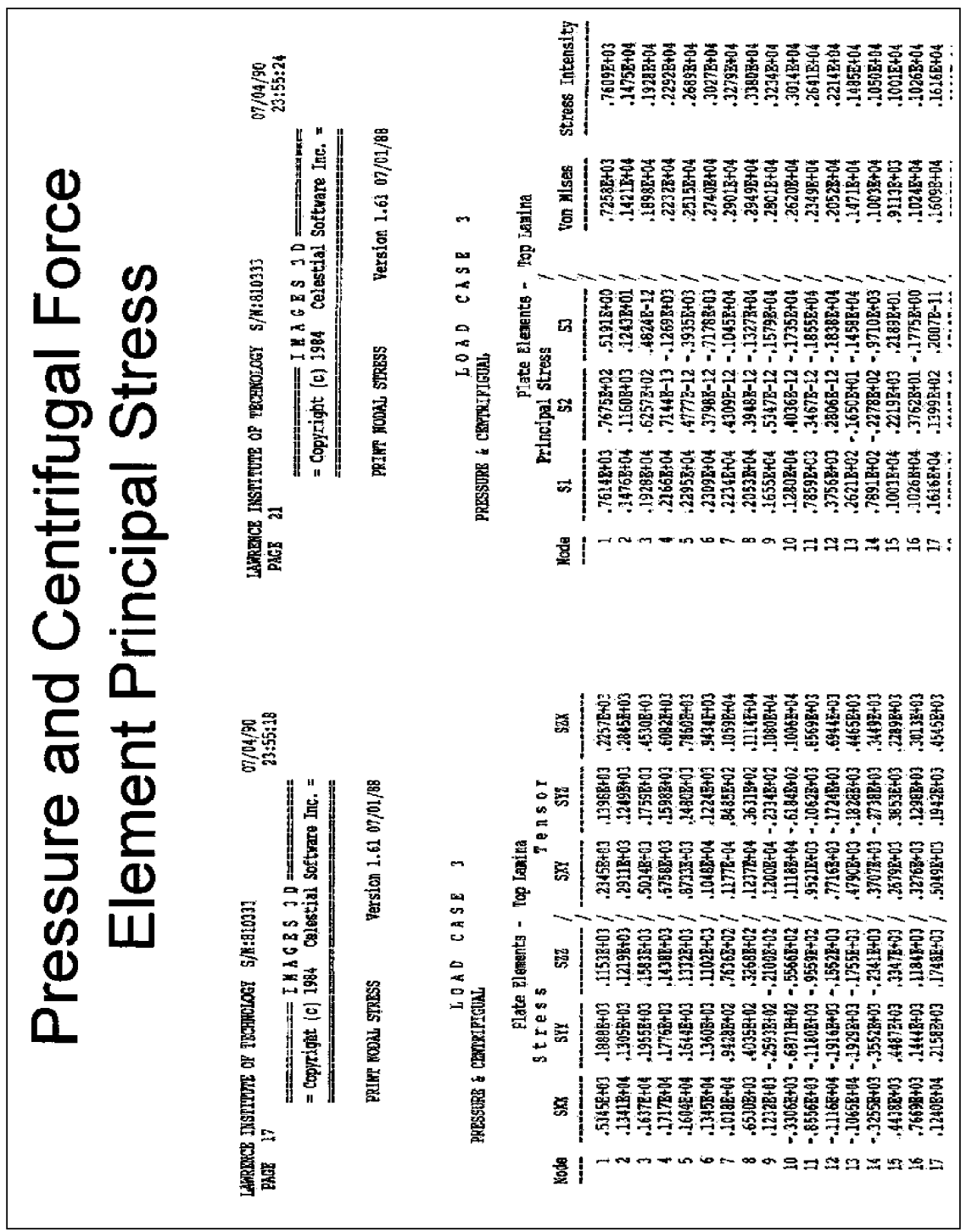


Copyright @ National Academy of Forensic Engineers (NAFE) http://www.nafe.org. Redistribution or resale is illegal. Originally published in the Journal of the NAFE volume indicated on the cover page. ISSN: 2379-3252

Analysis during the engine operating range revealed insufficient centrifugal force acting on the blades which would produce rivet overload stress and subsequent failure. Figure \#6 is a tabulation of the generated flex-fan blade centrifugal force, at various engine rpm. Flex-Fan blade natural frequencies were obtained which revealed dynamic blade flutter. Figure \#7 is a tabulation of these flex-fan blade natural frequencies. Photos \#15, \#16 and \#17 are flex-fan blade configurations during these natural frequencies.

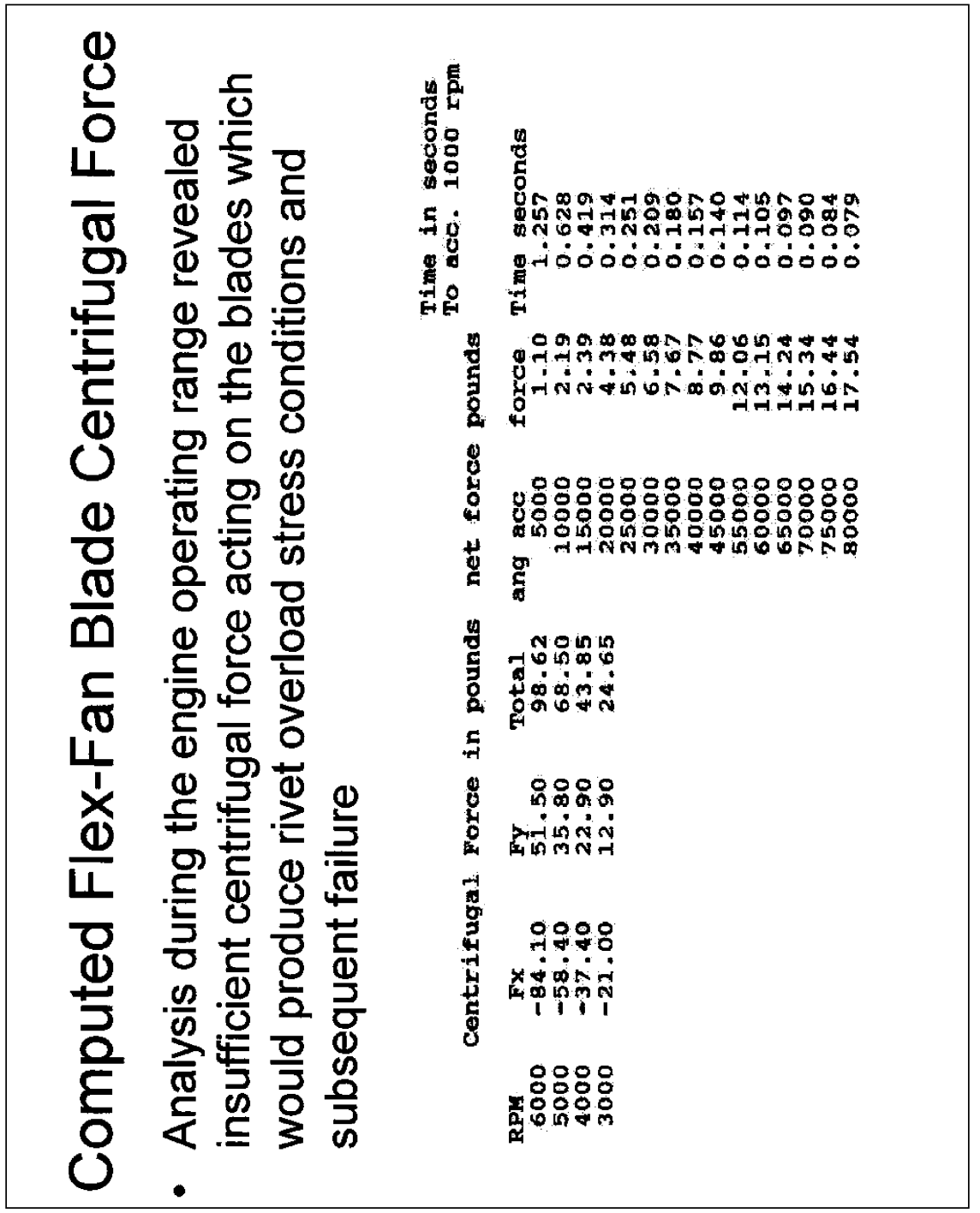

Figure 6 
Copyright $\odot$ National Academy of Forensic Engineers (NAFE) http://www.nafe.org. Redistribution or resale is illegal. Originally published in the Journal of the NAFE volume indicated on the cover page. ISSN: 2379-3252

Analysis of flex-fan blade flutter revealed compromising of rivet retention due to tensile induced loads and subsequent compromising of the retaining hub, permitting blade movement. Engine firing frequency creates a rotational fan assembly pulse consistent with the identified rivet chatter lines resulting in rivet failure and separation of the flex-fan blade from the companion hub.

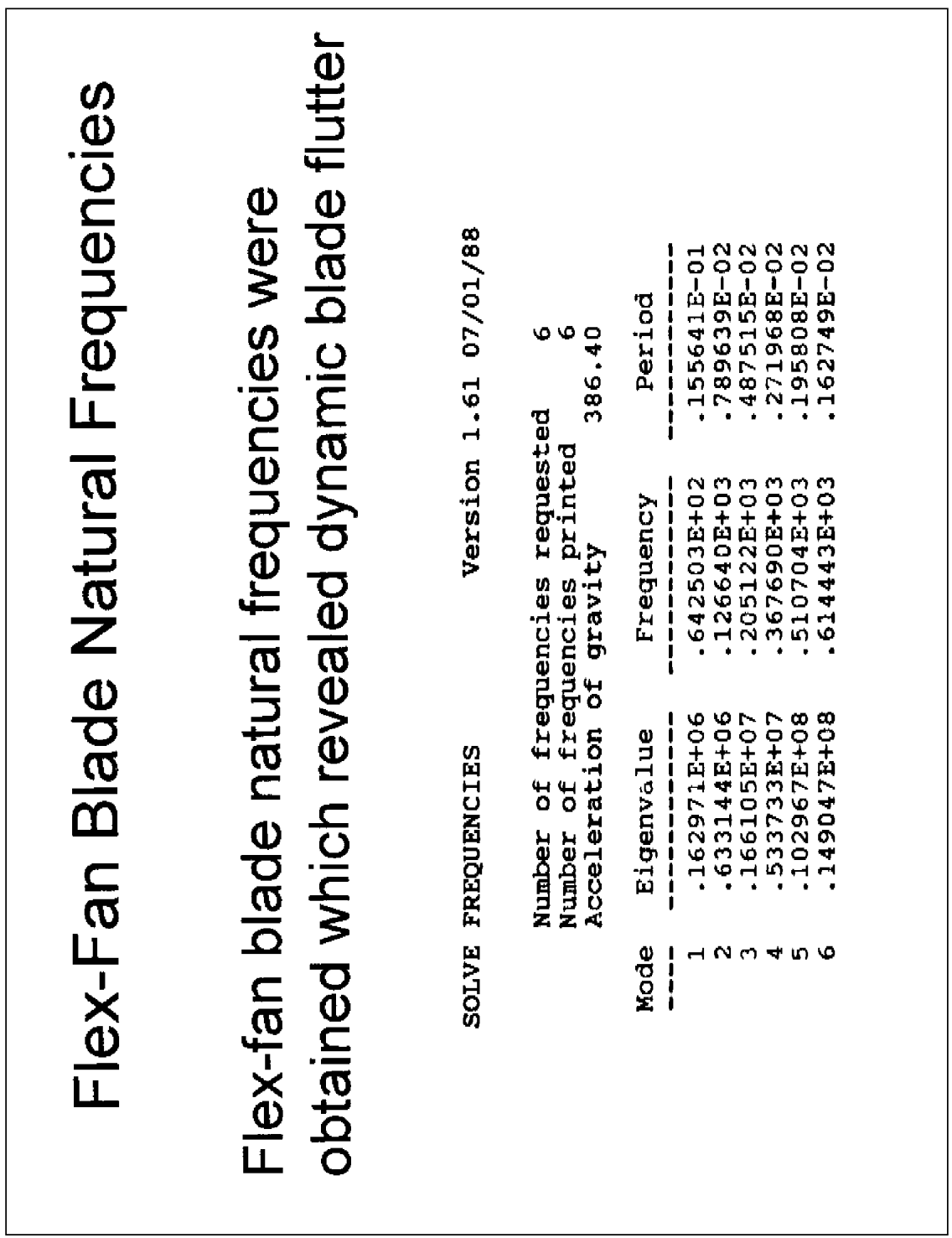


Copyright @ National Academy of Forensic Engineers (NAFE) http://www.nafe.org. Redistribution or resale is illegal. Originally published in the Journal of the NAFE volume indicated on the cover page. ISSN: 2379-3252

\section{Conclusion}

It is apparent, mechanical mechanisms experience dynamic loads not identified during the design process or pre production testing. Laboratory static tests frequently do not address anticipated dynamic loads and subsequent component stress distribution. Dynamic flex-fan blade deformation, stress magnitude and distribution experienced were identified using a finite element analysis. This analysis revealed flex-fan blade resonant frequencies, corresponding blade flutter and high concentrated stress areas within the expected engine operating range. Combined with the physical evidence provided by failed rivet fracture surfaces, metallurgical analysis and a finite element analysis the reconstruction

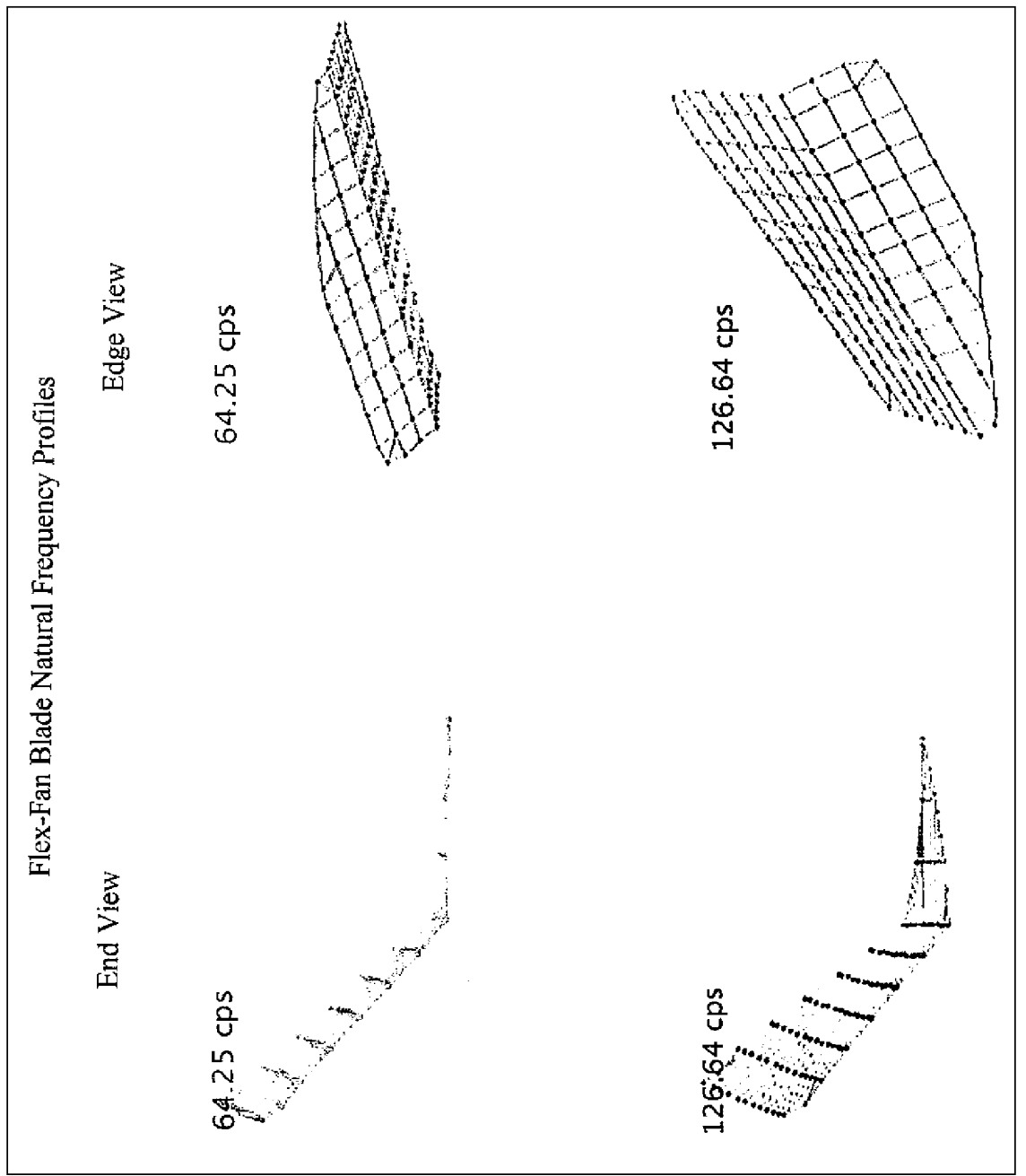


Copyright $\odot$ National Academy of Forensic Engineers (NAFE) http://www.nafe.org. Redistribution or resale is illegal. Originally published in the Journal of the NAFE volume indicated on the cover page. ISSN: 2379-3252

of flex-fan blade separations during a short operating period was determined. Application of finite element analysis as a design and/or Forensic Engineering reconstruction tool is essential in identifying failure modes and providing alternative solutions.

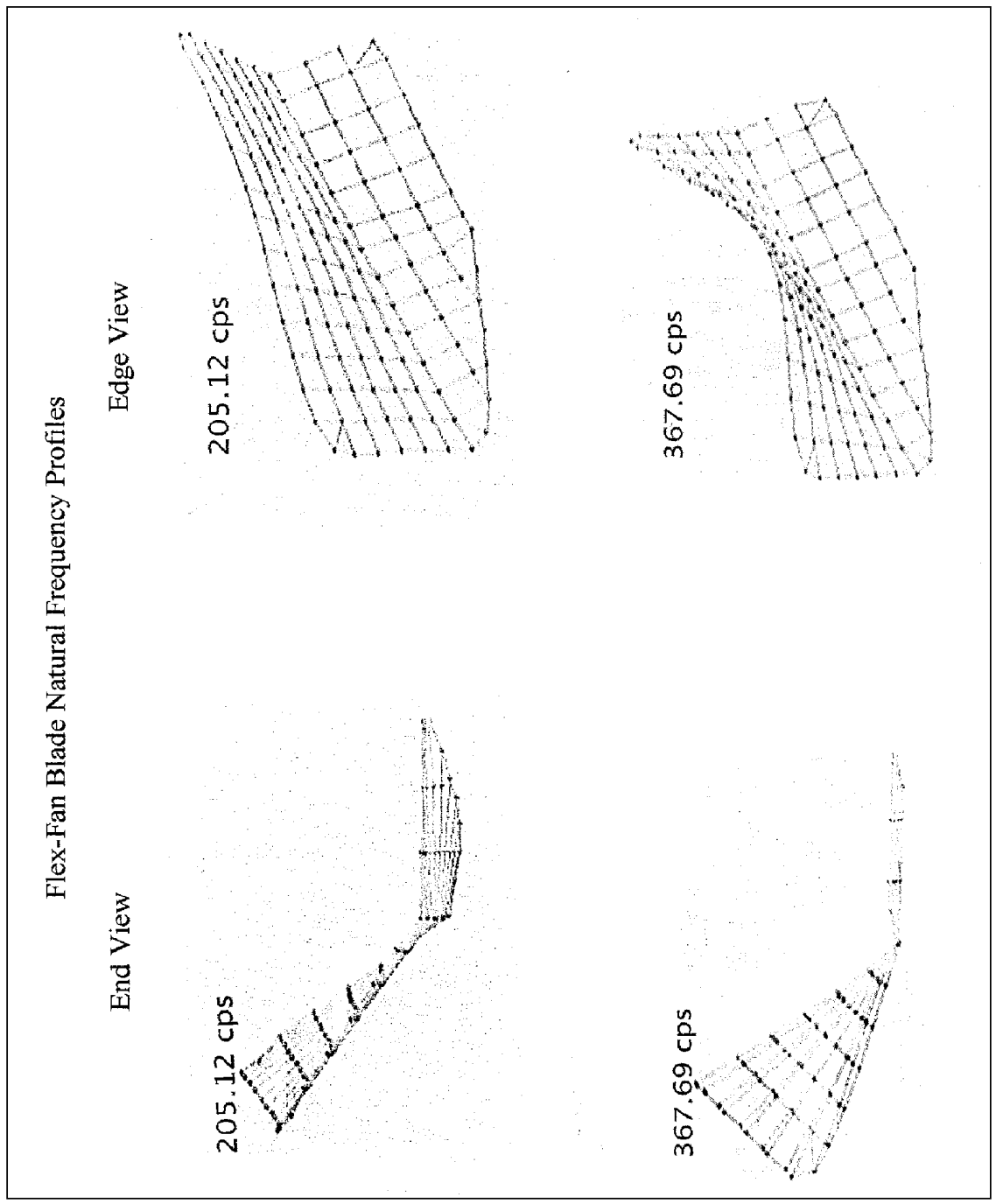

Photo 16 
Copyright @ National Academy of Forensic Engineers (NAFE) http://www.nafe.org. Redistribution or resale is illegal.

Originally published in the Journal of the NAFE volume indicated on the cover page. ISSN: 2379-3252

NAFE 205F FINITE ELEMENT ANALYSIS OF A FAN BLADE FAILURE

PAGE 17

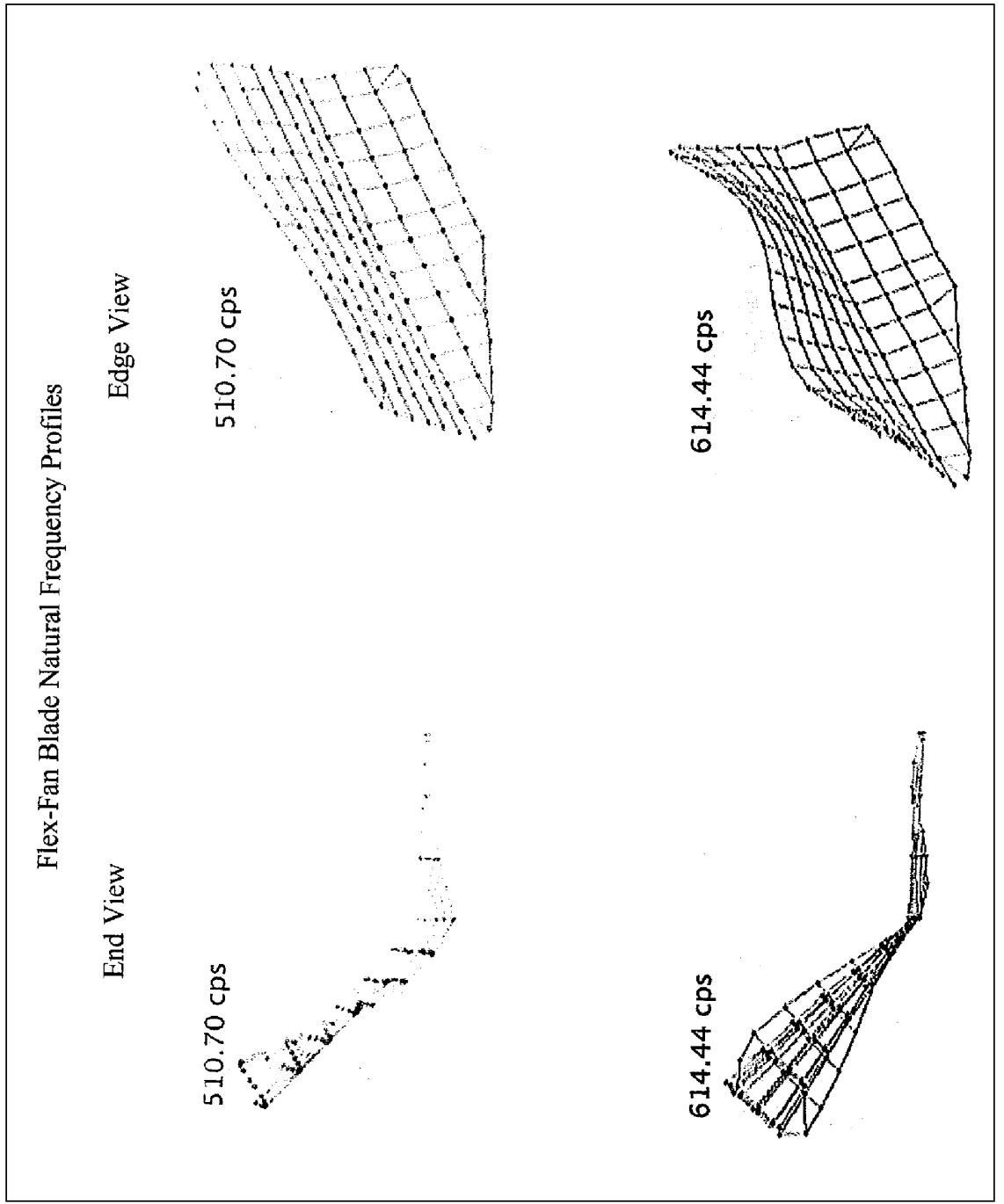

Photo 17 
Copyright $\odot$ National Academy of Forensic Engineers (NAFE) http://www.nafe.org. Redistribution or resale is illegal. Originally published in the Journal of the NAFE volume indicated on the cover page. ISSN: 2379-3252

\section{References}

1. Bowes, W. H. and Russell, L.T., Stress Analysis by the Finite Element Method for Practicing Engineers, Lexington Book, D.C. Health, Lexington, Mass. 1975

2. Dubinsky, R.G., "The Finite Element Method and Computer Aided Engineering-Users View," A.S.M.E. Design Conference, Session 2.1-Finite Element Method in Automotive Design, Chicago, April 27, 1981

3. Huebner, K.H., "The Finite Element Method for Engineers," John Wiley \& Sons, New York, 1975

4. Parker, G.R., "modeling Moving Parts with Dynamics FEM, "ComputerAided Engineering, October 1984

5. Bathe, K.J., "Finite Element Procedures in Engineering Analysis," Prentice Hall, Inc., Englewood Cliffs, New Jersey, 1981

6. Cheung, Y.K. and Yeo, M.F., "A Practical Introduction to Finite Element Analysis," Pitman Publishing, London, 1979 